\begin{abstract}
We propose a novel, biologically plausible cost/fitness function for sensorimotor control, formalized with the information-theoretic principle of empowerment, a task-independent universal utility. Empowerment captures uncertainty in the perception-action loop of different nature (e.g. noise, delays, etc.) in a single quantity. We present the formalism in a Fitts' law type goal-directed arm movement task and suggest that empowerment is one potential underlying determinant of movement trajectory planning in the presence of signal-dependent sensorimotor noise. Simulation results demonstrate the temporal relation of empowerment and various plausible control strategies for this specific task.
\end{abstract}




\title{
Information-theoretic Sensorimotor Foundations of Fitts' Law
}

\author{
Dari Trendafilov ${ }^{1} \quad$ Daniel Polani ${ }^{2}$ \\ ${ }^{1}$ Johannes Kepler University, Linz, Austria, \\ dari.trendafilov@pervasive.jku.at \\ ${ }^{2}$ University of Hertfordshire, Hatfield, UK, \\ d.polani@herts.ac.uk
}

\section{Introduction and Background}

Goal-directed arm movement entails the localization of the hand and the target, motor planning and execution. In all of these phases, neural signals are corrupted by noise imposing limits on human performance. Sensor noise leads to uncertainty about objects' position, degrading perception, while motor noise leads to loss in precision, resulting in movement inaccuracy and variability. It has been shown that the standard deviation in isometric force production is proportional to the mean force Schmidt et al., 1979, Meyer et al., 1988, consistent with psychophysical observations reflected by Fitts' law Fitts, 1954 - i.e. the variability of motor errors increases with the magnitude of movement. Since fast movements require large control signals, which increase the variability in the final position due to signal-dependent noise, this implies a trade-off between movement duration and terminal accuracy.

Motor planning determines the optimal actions based on signals from sensory inputs and motor outputs. The redundancy in the motor system allows for infinite possibilities for performing a specific movement, however, humans tend to produce stereotypical patterns, which suggests that movement control may have evolved so as to optimize a specific cost function subject to certain biophysical constraints Harris, 1998. For arm trajectories, which are typically smooth with approximately straight paths and bell-shaped velocity profiles Morasso, 1981, Kelso et al., 1979, it has been proposed that the cost function being optimized is the mean-squared jerk of the hand [Hogan, 1984, Flash and Hogan, 1985] or the mean-squared rate of change of the joint torques [Uno et al., 1989. Although these cost functions predict the observed movement well, they are heuristic in their nature and are hard to compute by the brain. An ideal cost would be universal for all systems (e.g. the eye and the arm alike), simple to compute, and have some identifiable evolutionary advantage. 

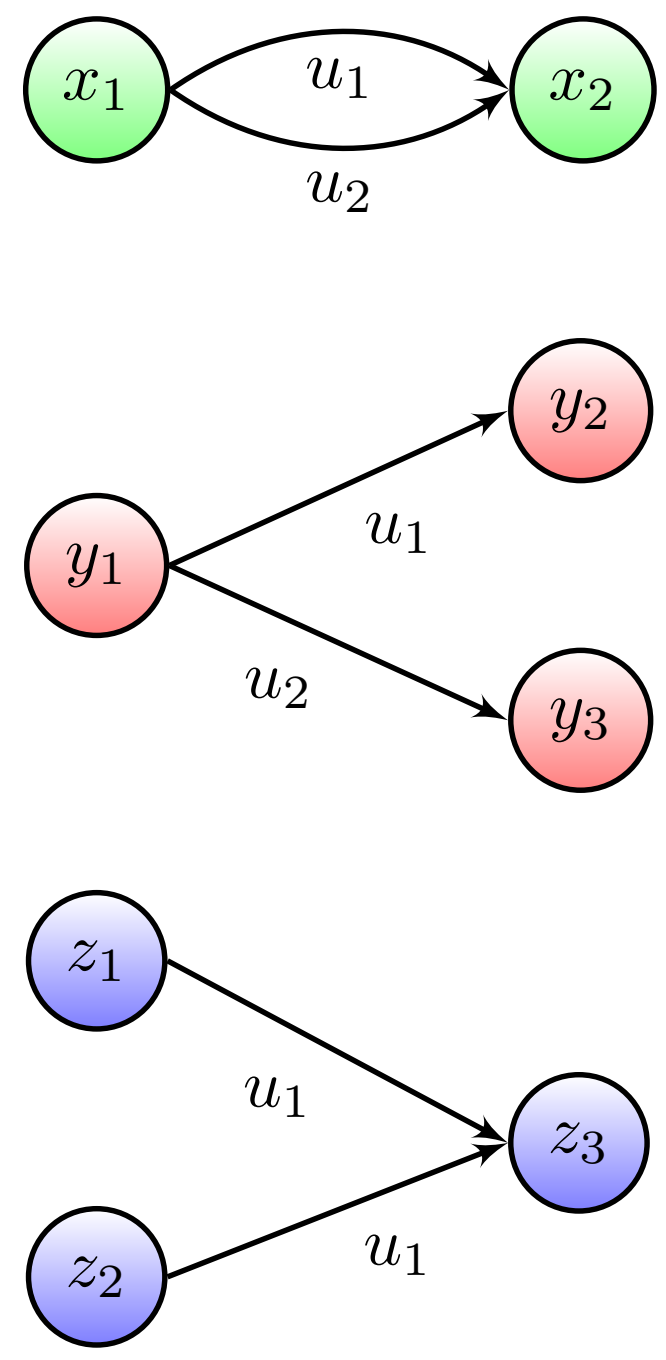

Figure 1: The graphs represent transitions between perception states $x, y, z$ when executing actions $u$. Intuitively, empowerment characterizes the number of actions available to the agent the outcome of which it can perceive. It is low if regardless of the action the perception will be the same (in green) and is high if every action implies a distinct perception (in red and blue). In the top (green) graph the agent has no real choice of an action as both outcomes yield the same perception state. In the middle and bottom graphs the empowerment in the initial nodes $\left(y_{1}, z_{1}\right.$ and $z_{2}$ respectively) is high as different actions lead to distinct outcomes. 
We propose that empowerment, a universal and generic information-theoretic utility measure introduced by Klyubin et al Klyubin et al., 2008, is one potential cost function humans optimize in sensorimotor control. The concept of empowerment is based on an information-theoretic model of the perceptionaction loop of an embodied agent and its environment. It provides a task- and representation-independent utility function that quantifies the maximum potential information flow from the agent's actuators to its sensors through the environment. Empowerment captures uncertainty of various sources (e.g. noise, delays, errors, etc.) in a generic theoretical measure, reflecting the level of perceivable control or influence an agent has over its environment (see Figure 1). Previously proposed cost functions Hogan, 1984, Flash and Hogan, 1985, Uno et al., 1989 imply smooth optimal arm trajectories, since abrupt changes would require large motor commands and thus higher noise, which supports the view of empowerment as a good candidate for providing a unified perspective of uncertainty inherent in sensorimotor control.

\section{Empowerment}

Empowerment is defined for stochastic dynamic systems (e.g. an agent interacting with an environment) in which transitions arise as the result of decisionmaking. It is fully specified by the dynamics of the agent-environment coupling and measures the amount of information an agent can inject into its environment and later perceive by its sensors, based on the channel capacity from the sequence of actions $U_{t-n}, U_{t-n+1}, \ldots, U_{t-1}$ to the perceptions $Y_{t}$ after an arbitrary number of time-steps

$$
C\left(U_{t-n}, \ldots, U_{t-1} \rightarrow Y_{t}\right)=\sup _{p(\vec{u})} I\left(U_{t-n}, \ldots, U_{t-1} ; Y_{t}\right)
$$

where $p(\vec{u})$ denotes the probability distribution of the action sequences (see Figure 22. For simplicity, we will assume full observability, where the agent perceives the true state of the world $\mathcal{X}$ and hence $\mathcal{X} \equiv \mathcal{Y}$. Given discrete state $\mathcal{X}$ and action $\mathcal{U}$ spaces, the transition function is given in terms of a density $p\left(x_{t+1} \mid x_{t}, u_{t}\right)$ reflecting the probability of moving from state $x_{t}$ to $x_{t+1}$ when selecting action $u_{t}$. Let $X^{\prime}$ denote the random variable associated with $x^{\prime}$ given $x$, and let the choice of a particular action $u$ be modelled by a random variable $U$. The empowerment $\mathfrak{E}(x)$ in state $x$ is defined as the Shannon channel capacity between the choice of an action sequence $U$ and the resulting successor state $X^{\prime}$, which depends on the temporal horizon

$$
\mathfrak{E}(x)=\max _{p(\vec{u} \mid x)} I\left(\mathcal{X}^{\prime} ; \mathcal{U} \mid x\right)=\max _{p(\vec{u} \mid x)}\left\{H\left(\mathcal{X}^{\prime} \mid x\right)-H\left(\mathcal{X}^{\prime} \mid \mathcal{U}, x\right)\right\} .
$$

The maximization of mutual information is with respect to all possible distributions over $\mathcal{U}$, where the entropies are given by $H\left(\mathcal{X}^{\prime} \mid x\right)=-\sum_{x^{\prime} \in \mathcal{X}} p\left(x^{\prime} \mid x\right) \log p\left(x^{\prime} \mid x\right)$ and $H\left(\mathcal{X}^{\prime} \mid \mathcal{U}, x\right)=-\sum_{\nu=1}^{N} p\left(u_{\nu}\right) H\left(\mathcal{X}^{\prime} \mid \mathcal{U}=u_{\nu}, x\right)=-\sum_{\nu=1}^{N} p\left(u_{\nu}\right) \sum_{x^{\prime} \in \mathcal{X}} p\left(x^{\prime} \mid x, u_{\nu}\right) \log p\left(x^{\prime} \mid x, u_{\nu}\right)$. 


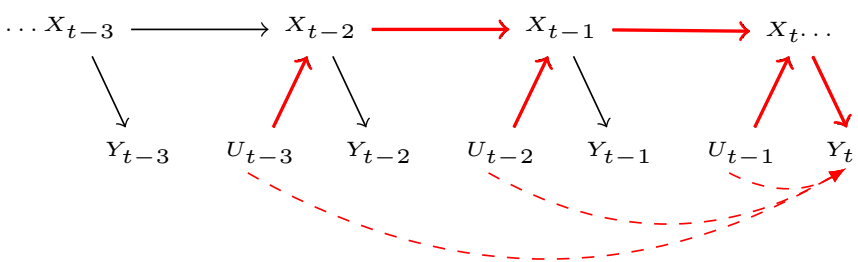

Figure 2: A causal Bayesian network representation of the perception-action loop unrolled in time, viewed as a communication channel - when the agent performs an action $U$, it injects information into the environment $X$ (following the red arcs), and subsequently reacquires part of this information from the environment via its sensors $Y$, where all elements are modelled as random variables. Empowerment captures the causal effect, which is a function of the agents embodiment, describing how the agents sensors and actuators interact with the environment.

Empowerment maximization enables the agent to benefit most from its sensors and actuators by keeping most of its options for influencing the environment open. Intuitively, empowerment measures on a logarithmic scale the number of actions available to the agent the outcome of which it can perceive. It is zero if regardless of the action the perception will be the same and is maximal if every action implies a distinct perception. Empowerment can be interpreted as the capacity of an agent to control or influence its environment as perceived by its sensors, i.e. it captures the uncertainty in agent's perceptions related to its actions.

\section{Control Model}

We computed empowerment in a goal-directed arm movement task using a linear model of the arm described by the following discrete-time system (some nonlinear models could be approximated too)

$$
x_{t+1}=A x_{t}+B u_{t}+w_{t},
$$

where $x_{t}$ is a 2-dimensional state (arm's linear velocity and acceleration) and $u_{t}$ is the control input (jerk) at time $t$. Since our model assumes positioninvariance of empowerment, for brevity, this property is not included in the state space. $A$ is a $2 \times 2$ system transfer matrix and $B$ a $2 \times 1$ vector describing the dynamics of the system. In order to demonstrate the trade-off between different sources of noise we assumed two components of $w_{t}$, a linear Gaussian noise on velocity $\mathcal{N}\left(0, k_{1} x_{t, 1}\right)$ and a quadratic Gaussian noise on jerk $\mathcal{N}\left(0, k_{2} u_{t, 2}^{2}\right)$, following evidence of a quadratic relationship between standard deviation of signal-dependent noise and magnitude of control Harris and Wolpert, 1998. Following the control model of Equation 2 we consider decision-making (i.e. 
motor planning) a continuous closed-loop feedback process, where, for simplicity, we ignore delays inherent in the perception-action loop, which are addressed in a separate study Trendafilov and Murray-Smith, 2013.

\section{Simulations}

Using the above model, we performed series of simulations with various system's parameters $A$ and $B$, and noise levels $w$. Based on simulated stochastic transition functions we evaluated 1-step empowerment in a discrete 2-D grid as a function of velocity and acceleration. Figure 3 presents the empowerment landscape of one simulation run in which, as expected, empowerment decreases from its peak at rest point with increasing velocity and acceleration, as agent's perception related to its actions deteriorates due to signal-dependent noise. For this particular set of parameters acceleration has a stronger impact on empowerment than velocity (i.e. imposes a higher noise penalty), reinforced by the quadratic term. The model demonstrates how two types of disturbances in the sensorimotor loop can be combined in a single generic measure of uncertainty. The empowerment landscape is not symmetric along the acceleration axis due to a trade-off between acceleration and velocity. This is particularly visible at high velocity where negative acceleration yields higher empowerment than the corresponding levels of positive acceleration.

From the landscape in Figure 3 we computed the average empowerment $\left(\frac{1}{T} \sum \mathfrak{E}_{t}\right)$ over movement trajectories corresponding to the four bell-shaped velocity profiles shown in Figure 4/b. The respective acceleration profiles (see Figure $4 / \mathrm{c}$ ) were selected from the family of Clausen functions defined by

$$
C l_{n}(\varphi)=\sum_{k=1}^{\infty} \frac{\sin k \varphi}{k^{n}}
$$

and achieving identical terminal accuracy $(16 \mathrm{~cm})$ and movement time (200ms) (see Figure 4/a). Figure 4/d presents running and average empowerment (solid and dashed lines) corresponding to different control strategies, indicating that $C l_{1.3}$ yields the highest average empowerment $(0.94 \mathrm{bits})$, while sine wave - the lowest (0.82 bits). Figure $4 / \mathrm{bc}$ reveals how larger initial control of $C l_{1.3}$ helps containing acceleration within $[-20,20] \mathrm{m} / \mathrm{sec}^{2}$ and velocity below $1.3 \mathrm{~m} / \mathrm{sec}$, while more sluggish control profiles require higher acceleration (up to $25 \mathrm{~m} / \mathrm{sec}^{2}$ ) and velocity (up to $1.6 \mathrm{~m} / \mathrm{sec}$ ).

Empowerment is highest at arm rest, as the agent can perceive best the outcome of its actions the least the noise. With acceleration and velocity (and noise) increase empowerment drops rapidly due to the loss of precision in perceiving the outcome of agent's actions. Empowerment reaches a local maxima at zero acceleration and maximal velocity for $C l_{1.3}$ and $C l_{1.5}$ as the agent's perception is most impaired by the quadratic penalty on jerk noise. For $\mathrm{Cl}_{2}$ and sine wave this local maxima is offset due to higher maximal velocities and acceleration/velocity trade-off. 


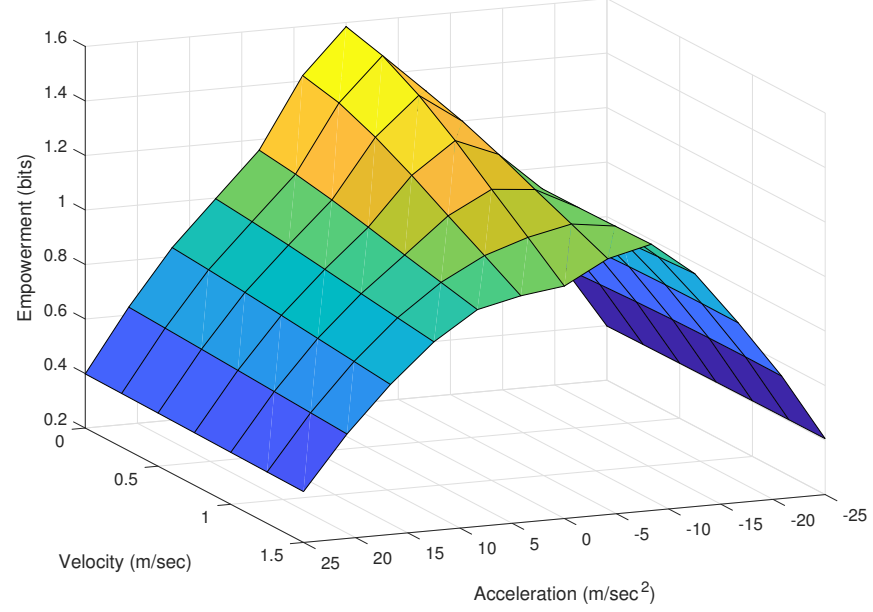

Figure 3: Empowerment landscape reflecting a particular set of model parameters evaluated in a discrete grid as a function of velocity and acceleration. Empowerment peaks at rest point, as the agent can perceive best the outcome of its actions the least the noise and gradually decreases with increasing magnitude of control as agent's perception deteriorates due to signal-dependent noise. Note that the empowerment profile is not symmetric along the acceleration axis. 
The empowerment trajectories have quasi symmetric shapes, except around local maxima, where non-symmetric empowerment landscapes at high velocities induce minor deviations (see Figure 3). Although characterized by different empowerment profiles, $C l_{1.3}$ and $C l_{1.5}$ achieve similar levels of average empowerment (0.94 vs. 0.93 bits), suggesting that empowerment-optimal control strategies might not be unique, which is an interesting topic for future investigation.

\section{DISCUSSION}

The control model investigated in this paper assumes a hypothetical set of parameters, such as noise levels and system transfer functions, for the sake of argument. Furthermore, as an evaluation criterion empowerment mean was chosen over more complex functionals, which could eventually provide different shapes. Such properties characterising human sensorimotor control are not completely understood yet and require further investigation. The evaluated four bell-shaped velocity profiles were selected so as to reveal how variations in control strategy affect empowerment. In this paper, we propose that in the presence of signal-dependent noise humans might select the velocity profile that maximizes empowerment subject to the specific model parameters discussed above.

Simulation results demonstrate that empowerment could provide a universal and generic measure of uncertainty in sensorimotor control tasks, such as goaldirected arm movement. In this context, empowerment maximization subject to internal signal-dependent noise provides a biologically plausible explanation for the relevance of previously proposed cost functions, such as jerk and torque change. However, the importance of empowerment for HCI research in general goes beyond this. Fitts' law has been studied extensively in a range of scenarios including hand-held devices where external sources of noise abound. Understanding how different internal (sensorimotor) and external (environmental) sources of noise interact with each other and how they influence uncertainty and bound human performance is particularly important in mobile contexts where noise is inevitable and often unpredictable.

Fast point-to-point movement studied with regard to Fitts' law is typically considered open-loop control, however we treat it as a closed-loop perceptionaction process, which is supported by recent empirical evidence [Müller et al., 2017. A number of studies on human sensorimotor control identify mechanisms of Bayesian inference when we deal with uncertainty Körding and Wolpert, 2006. A recent study proposed an information-theoretic characterisation of variance in aimed movement with feedback using Shannon channel capacity Gori and Olivier, 2018.

The proposed approach has several important ramifications. Empowerment is conceptually a new quantity in HCI research, which combines different types of noise in a single theoretical measure. It provides a biologically plausible theoretical underpinning for estimating the cost of arm movements, captured by a universal and generic measure of control. A cost function, based solely on 
a

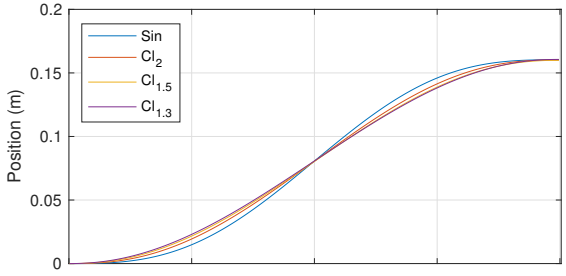

b

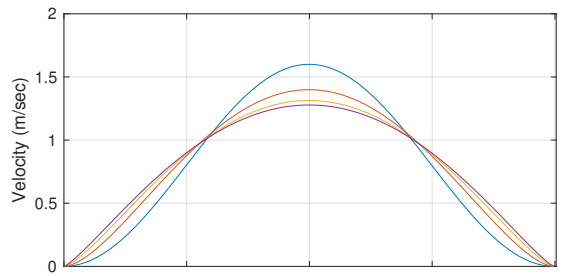

c

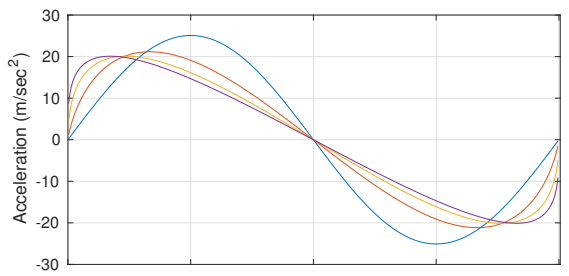

d

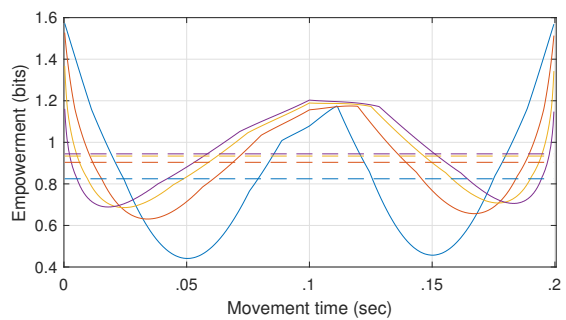

Figure 4: a) Position, b) velocity, c) acceleration, and d) empowerment series. For given acceleration profiles (sine wave and three Clausen functions) velocity and position are integrated. Movement time and terminal accuracy are aligned to $200 \mathrm{~ms}$ and $16 \mathrm{~cm}$, respectively. Empowerment levels (solid lines) correspond to values in Figure $3 . C l_{1.3}$ achieves highest average empowerment (0.94 bits), followed by $C l_{1.5}$ (0.93 bits) (dashed lines). 
uncertainty, such as empowerment, is directly available to the human brain via feedback [Meyer et al., 1988. Furthermore, the resulting optimal trajectories would be inherently smooth, since abrupt changes would require large driving signals, implying higher noise levels and degrading agent's perception of the outcome of its actions, leading to lower empowerment.

\section{Conclusion}

In this paper we propose that the information-theoretic principle of empowerment could provide a biologically plausible generic and universal cost/fitness function for sensorimotor control. As an analytical measure it could explain and predict human performance in the presence of stochastic disturbances and serve as an objective optimality criterion in UI optimization as suggested in Trendafilov et al., 2015, trading off diverse sources of uncertainty. Validating the approach in user studies is an exciting topic for future research. The aim of this paper is to raise the awareness of the HCI community about the potential empowerment has in providing more solid theoretical foundations for the science of HCI.

\section{Acknowledgment}

We acknowledge support by H2020-641321 socSMCs FET Proactive and FFG6112792 Pro $^{2}$ Future.

\section{References}

[Fitts, 1954] Fitts, P. M. (1954). The information capacity of the human motor system in controlling the amplitude of movements. J. Exp. Psychol., 47:381391.

[Flash and Hogan, 1985] Flash, T. and Hogan, N. (1985). The coordination of arm movements: an experimentally confirmed model. J. Neurosci., 5:16881703.

[Gori and Olivier, 2018] Gori, J. and Olivier, R. (2018). Information-theoretic analysis of the speed-accuracy tradeoff with feedback. IEEE International Conference on Systems, Man, and Cybernetics (SMC), pages 3452-3457.

[Harris, 1998] Harris, C. M. (1998). On the optimal control of behaviour: a stochastic perspective. J. Neurosci. Meth., 83:73-88.

[Harris and Wolpert, 1998] Harris, C. M. and Wolpert, D. M. (1998). Signaldependent noise determines motor planning. Nature, 394:780-784.

[Hogan, 1984] Hogan, N. (1984). An organizing principle for a class of voluntary movements. J. Neurosci., 4:2745-2754. 
[Kelso et al., 1979] Kelso, J. A. S., Southard, D. L., and Goodman, D. (1979). On the nature of human interlimb coordination. Science, 203:1029-1031.

[Klyubin et al., 2008] Klyubin, A. S., Polani, D., and Nehaniv, C. L. (2008). Keep your options open: An information-based driving principle for sensorimotor systems. PLoS ONE, 3(12).

[Körding and Wolpert, 2006] Körding, K. and Wolpert, D. (2006). Bayesian decision theory in sensorimotor control. Trends in Cog. Sci., 10:319-326.

[Meyer et al., 1988] Meyer, D. E., Abrams, R. A., Kornblum, S., Wright, C. E., and Smith, J. E. K. (1988). Optimality in human motor performance: ideal control of rapid aimed movements. Psychol. Rev., 98:340-370.

[Morasso, 1981] Morasso, P. (1981). Spatial control of arm movements. Exp. Brain Res., 42:223-227.

[Müller et al., 2017] Müller, J., Oulasvirta, A., and Murray-Smith, R. (2017). Control theoretic models of pointing. ACM Transactions on ComputerHuman Interaction, 24(4):27.

[Schmidt et al., 1979] Schmidt, R. A., Zelaznik, H., Hawkin, B., Frank, J. S., and Quinn, J. T. (1979). Motor-output variability: a theory for the accuracy of rapid motor acts. Psychol. Rev., 86:415-451.

[Trendafilov and Murray-Smith, 2013] Trendafilov, D. and Murray-Smith, R. (2013). Information- theoretic characterization of uncertainty in manual control. In Proc. IEEE SMC conference.

[Trendafilov et al., 2015] Trendafilov, D., Murray-Smith, R., and Polani, D. (2015). Empowerment as a metric for optimization in hci. CHI 2015 Workshop on Principles, Techniques and Perspectives on Optimization and HCI.

[Uno et al., 1989] Uno, Y., Kawato, M., and Suzuki, R. (1989). Formation and control of optimal trajectories in human multijoint arm movements: minimum torque-change model. Biol. Cybern., 61:89-101. 